\title{
Iranian drug use survey in workplaces: A study protocol
}

\author{
Behzad Damari ${ }^{1}$, Hossein Almadani², Mahin Ahmadi Pishkuhi*3
}

Received: 10 Feb 2018

Published: 29 Sep 2018

\begin{abstract}
Background: Addiction is a serious concern in workplaces, as it can lead to accidents, absenteeism, and loss of productivity, neglect, delay, arguing, unhappiness, production cuts, and irregular working pattern. This national survey was conducted to assess the prevalence and pattern of drug use among employees of industrial environments in Iran.

Methods: In this cross sectional study, the sample size was determined to be 13489 using multistage cluster sampling method during November 2013 and November 2014. Data were collected by trained interviewers using a structured questionnaire developed by the research team. Urine drug test was done for participants using laboratory rapid kits. A total of 13286 questionnaires were completed with remarkable responsiveness such that only $1.2 \%$ of them were excluded due to defects in urine drug tests, and the rest were analyzed after the review.

Results: The response rate to the questionnaires was $98.4 \%$ and 13128 individuals took the urinary drug test. Many participants who had positive urine test did not express drug use and positive tests were more than self-reported cases. Compared to information about the pattern of drug use, data on the estimated prevalence of drug use was more reliable.

Conclusion: This study presents the protocol of a well-designed national survey, including sampling procedure, appropriate instrument and test, field work, and discussion on the strengths and limitations of the study, and thus its results can be used effectively by policymakers.
\end{abstract}

Keywords: Drug use, Survey, Workplace, Addiction

Conflicts of Interest: None declared

Funding: Research and Education Office of Headquarters for Combating Drugs, Tehran, Iran

\section{*This work has been published under CC BY-NC-SA 1.0 license. \\ Copyright $\odot$ Iran University of Medical Sciences}

Cite this article as: Damari B, Almadani H, Ahmadi Pishkuhi M. Iranian drug use survey in workplaces: A study protocol. Med J Islam Repub Iran. 2018 (29 Sep);32:93. https://doi.org/10.14196/mjiri.32.93

\section{Introduction}

Drug addiction is considered as one of the global crises of the third millennium, and drug use is one of the most important problems of humans which exposes the family and friends of the drug addicts to its devastating effects (1, 2 ). At the same time, addiction causes social hazards, such as the increased risks of divorce, increment in marriage age, and capital reduction (3).

In Iran, similar to other countries, the number of drug users is increasing and the age of onset of drug use is reducing (4). There are about 3 million injected drug users

Corresponding author: Dr Mahin Ahmadi Pishkuhi, mahin.ahmadipishkuhi@gmail.com

1. Tehran University of Medical Sciences, Neuroscience Institute, Governance and Health Department, Tehran, Iran

2. Department of Prevention of Social Pathologies, Ministry of Co-operation, Work and Social Welfare, Tehran, Iran

3. Pars Advanced and Minimally Invasive Medical Manners Research Center, Pars Hospital, Iran University of Medical Sciences, Tehran, Iran in the country, most of whom are HIV-infected, which is one of the negative indices in the country (5). The prevalence surveys in Iran indicate that possibly $8.2 \%$ of the 15-64 year- old population in the country are drug users (6); however, the accuracy of this prevalence estimate is unclear because of social stigma and legal restrictions of drug use in Iran (7).

The global phenomenon of increasing prevalence of drug use in industrial environments is accounted as a serious hazard, as workers have an important role in

$\uparrow$ What is "already known" in this topic:

Identifying the prevalence of psychotropic substances and drug use in workplaces can be effective for political directors to develop preventive and treatment programs.

\section{$\rightarrow$ What this article adds:}

The present protocol has a reliable methodology to run periodical national surveys of prevalence of addiction in Iran's industrial environments. Also, it can be used as a pattern for designing other surveys with similar aims at the national level. 
development of the economy in communities and as drug depresses labor productivity, increases absenteeism, work accidents, and injuries (8). Addiction rates in Asalouyeh, as a major industrial hub of Iran, have been estimated to be $15 \%-25 \%$ (9). However, the observed bias and selection bias were not controlled in the survey.

Political directors should have sufficient knowledge about the magnitude of problem of addiction in the community workplaces to plan for intervention, design, and development of drug prevention and treatment programs. Nevertheless, access to accurate statistics and information in this area was still difficult due to the inconsistency among references and researches about the prevalence of drug use $(10,11)$.

National survey of drug use and addiction among employees of Iran industrial centers was performed by the Ministry of Cooperatives, Labor and Social Welfare and Office of Research and Training Center for Combating drug. Iranian drug use in workplaces survey (IDUWS) was conducted to determine the regions with most prevalent use of drugs and psychoactive substances. Also, prevalence estimation and patterns of drug use in each area were assessed. The protocol of the investigation was described as an appropriate structural model for Iran.

The main objectives of the project were as follow:

- Estimating the prevalence of psychotropic substances and drug use according to usage pattern in 1 week, 1 month, and 1 year ago, and the age of first use among employees of the industrial centers in Iran
- Determining the high-risk areas of drug consumption in the country based on drug and psychotropic substances type among the staffs of industrialized centers

- Identifying patterns of drug use among employees of industrial centers in the country

- Evaluating drug use and psychoactive substances based on demographic characteristics, the consumption pattern of first use, type of drug, frequency of consumption, present consumption patterns, drug injection, smoking, alcohol consumption, withdrawal, treatment, arrest, and imprisonment history.

\section{Methods}

\section{Study design}

National Survey of Drug Addiction Prevalence in the Workplace of Iran was a descriptive cross sectional survey that was conducted during November 2013 and November 2014 in 2 phases. The first phase was collecting data through a self-reported questionnaire (data collection through interviews by trained interviewers based on a structured questionnaire), and the second phase was verification with urine tests.

\section{Study population, sample size, and sampling}

The target population consisted of all employees of industrial centers in all provinces of the country. The sample size was 6200, which was calculated by multiplying the number of provinces by the number of sample size in each province (31 provinces* at least 200

Table 1. The percentage of sample size in different provinces of Iran

\begin{tabular}{|c|c|c|c|c|c|c|}
\hline Province & $\begin{array}{c}\text { Number of } \\
\text { workers in } \\
\text { each province }\end{array}$ & $\begin{array}{l}\text { percentage of number } \\
\text { of workers in each } \\
\text { province to total }\end{array}$ & $\begin{array}{l}\text { Percentage } \\
\text { of sample } \\
\text { size }\end{array}$ & $\begin{array}{l}\text { Sample } \\
\text { size }\end{array}$ & $\begin{array}{l}\text { The adjusted } \\
\text { sample size }\end{array}$ & $\begin{array}{c}\text { Percentage of the } \\
\text { adjusted sample } \\
\text { size }\end{array}$ \\
\hline East Azarbaijan & 68,120 & 5.2 & 5.2 & 622 & 622 & 4.6 \\
\hline West Azarbaijan & 18,090 & 1.4 & 1.4 & 165 & 200 & 1.5 \\
\hline Ardebil & 8,955 & 0.7 & 0.7 & 82 & 200 & 1.5 \\
\hline Isfahan & 141,145 & 10.7 & 10.7 & 1,290 & 1,290 & 9.6 \\
\hline Alborz & 75,700 & 5.8 & 5.8 & 692 & 692 & 5.1 \\
\hline Ilam & 1,720 & 0.1 & 0.1 & 16 & 200 & 1.5 \\
\hline Bushehr & 18,275 & 1.4 & 1.4 & 167 & 200 & 1.5 \\
\hline Tehran & 258,535 & 19.7 & 19.7 & 2,362 & 2,362 & 17.5 \\
\hline Chaharmahal and Bakhtiari & 12,235 & 0.9 & 0.9 & 112 & 200 & 1.5 \\
\hline South khorasan & 6,930 & 0.5 & 0.5 & 63 & 200 & 1.5 \\
\hline Razavi Khorasan & 95,905 & 7.3 & 7.3 & 876 & 876 & 6.5 \\
\hline North Khorasan & 8,760 & 0.7 & 0.7 & 80 & 200 & 1.5 \\
\hline Khuzestan & 65,390 & 5.0 & 5.0 & 598 & 598 & 4.4 \\
\hline Zanjan & 29,455 & 2.2 & 2.2 & 269 & 269 & 2.0 \\
\hline Semnan & 34,800 & 2.6 & 2.6 & 318 & 318 & 2.4 \\
\hline Sistan and Baluchestan & 3,620 & 0.3 & 0.3 & 33 & 200 & 1.5 \\
\hline Fars & 46,360 & 3.5 & 3.5 & 424 & 424 & 3.1 \\
\hline Qazvin & 79,255 & 6.0 & 6.0 & 724 & 724 & 5.4 \\
\hline Qom & 26,190 & 2.0 & 2.0 & 239 & 239 & 1.8 \\
\hline Kordestan & 5,795 & 0.4 & 0.4 & 53 & 200 & 1.5 \\
\hline Kerman & 21,475 & 1.6 & 1.6 & 196 & 200 & 1.5 \\
\hline Kermanshah & 16,055 & 1.2 & 1.2 & 147 & 200 & 1.5 \\
\hline Kohgiluyeh and Boyer-Ahmad & 2,980 & 0.2 & 0.2 & 27 & 200 & 1.5 \\
\hline Golestan & 16,835 & 1.3 & 1.3 & 154 & 200 & 1.5 \\
\hline Gilan & 39,410 & 3.0 & 3.0 & 360 & 360 & 2.7 \\
\hline Lorestan & 10,940 & 0.8 & 0.8 & 100 & 200 & 1.5 \\
\hline Mazandaran & 51,630 & 3.9 & 3.9 & 472 & 472 & 3.5 \\
\hline Markazi & 71,040 & 5.4 & 5.4 & 649 & 649 & 4.8 \\
\hline Hormozgan & 16,195 & 1.2 & 1.2 & 148 & 200 & 1.5 \\
\hline Hamedan & 18,320 & 1.4 & 1.4 & 167 & 200 & 1.5 \\
\hline Yazd & 43,105 & 3.3 & 3.3 & 394 & 394 & 2.9 \\
\hline total & $1,313,220$ & 100.0 & 100.0 & 12,000 & 13,489 & 100.0 \\
\hline
\end{tabular}


Table 2. Percentage and number of sample sizes according to type of workshops

\begin{tabular}{|c|c|c|c|c|}
\hline \multirow[t]{2}{*}{ Type of workshops } & \multicolumn{2}{|c|}{ Total number of workforces } & \multicolumn{2}{|c|}{ Sample size } \\
\hline & Number & Percentage & Number & Percentage \\
\hline Workshops with 10-49 employees & 246,620 & 18.8 & 2,533 & 18.8 \\
\hline Workshops with 50-99 employees & 160,350 & 12.2 & 1,647 & 12.2 \\
\hline Workshops with 100-499 employees & 498,750 & 38.0 & 5,123 & 38.0 \\
\hline Workshops with 500-999 employees & 169,500 & 12.9 & 1,741 & 12.9 \\
\hline Workshops with 1000 or more employees & 238,000 & 18.1 & 2,445 & 18.1 \\
\hline
\end{tabular}

participants in each province $=6200$ ). A prevalence of $10 \%$ and $4 \%$ precision were considered to calculate the least needed sample size in each province $(200$ individuals). Then, the calculated sample size (6200) was multiplied by the design effect of 1.9 to consider the clustering effects. The number of samples was estimated 12000 in this stage. In the next stage, the number of samples was adjusted and divided by the anticipated response rate of $88 \%$. Finally, the overall sample size was estimated to be 13489 , and the sample size in each province was calculated in proportion to size based on the percentage of workers in each province (Table 1). A list of workshops as sampling units was prepared and arranged according to the number of activists and was categorized based on the active population. The proportion of workers population in each category to the country's total worker population was calculated and attempted to be consistent with the percent of sample size in each group (Table 2).

\section{Sampling process}

Workshops with fewer than 10 active employees were excluded from the study because of the lack of information. Workshops with 10 to 50 active employees were studied. All participants were studied. Workshops with more than 50 active employees were classified into groups of workshops with 50 to 99 people, 100 to 500 , 501 to 1000 , and more than 1000 . Twenty workers were selected from the list of eligible people in each workshop using the table of random numbers and were studied. Sampling from workshops was done based on the list announced to the provinces. If it was not possible for any reason (revocation of the workshop, displacement, and in case an address was not found, etc.), new workshops were randomly substituted, considering the number of employees in each workshop category. Tofigh-Daru, a pharmaceutical company in Mashhad, was selected to implement the pilot phase.

\section{Field work organization}

The Office of Research and Education of Headquarters for Combating Drugs had proposed the importance and necessity of this investigation and implemented this survey in partnership with Cooperatives, Labor, and Social Welfare Ministry. Moreover, General Directors of Cooperatives, Labor and Social Welfare offices in the provinces were invited to the ministry and the necessity of performing this survey was explained to them. Then, the Offices of Cooperative, Labor, and Social Welfare were asked to select their interviewers in each province.

Interviewers and Training of the interviewers

Among the experts of the Labor Department who announced their willingness to participate in questioning, 37 male and 29 female interviewers were selected at the provincial level. The interviewers' selection criteria were as follow: being familiar with the research and questioning, and having work experience in the field of addiction. The interviewers were trained to discriminate between different drug types and to be acquainted with questioning protocol (ie, sampling method according to the industrial environments and the names of the companies surveyed, the provincial sample size, and the guidelines for complementation of the questionnaires). The interviewers were also instructed to perform laboratory tests and complete the questionnaires. After checking for the completeness of the questionnaires, those interviewers who did not have enough precision in completing the forms were notified about the necessary comments over the phone (Fig. 1). At the end of the project, those provincial interviewers who had a good performance were admired and appreciated by the General Director of the Social Affairs from Ministry of Cooperatives, Labor, and Social Welfare.

\section{Fieldwork procedures}

The General Director of Cooperatives, Labor, and Social Welfare Office in each province had mailed a letter to the managers of economic institutions to inform them about the project of drug use prevalence in workplaces, attracting interviewers' collaboration with researchers. The managers were supposed to inform their staff about the issue. The interviewers started with showing their inquiry cards to the employers of the workshops or industrial environments and presented a complete description about the project. Then, with the coordination of the employer, an approximately large hall or room beside the toilets was specified for interviews, and a safe place was determined to put urine samples. Participants were invited to the room in groups of 10 based on the random list that was set previously. Interviewers were cautious that the employer had not made a mistake in the random selection of the cases and had not selected only the addicted individuals. Before the interview, tea and other fluids were served for the interviewers, and all other staffs had to go out of the room at the time of the interview. Aims and the missions of this research were explained to each individual and they were notified about the significance of the results and respecting anonymity. Each individual was given a number from 1 to 10 . The same numbers were considered for questionnaires and disposable cups. Questioning was conducted with colleagues simultaneously based on guidelines. Then, participants were asked to do the urine test voluntarily. Urine tests for males was supervised by a male and those for females was observed by a female. 


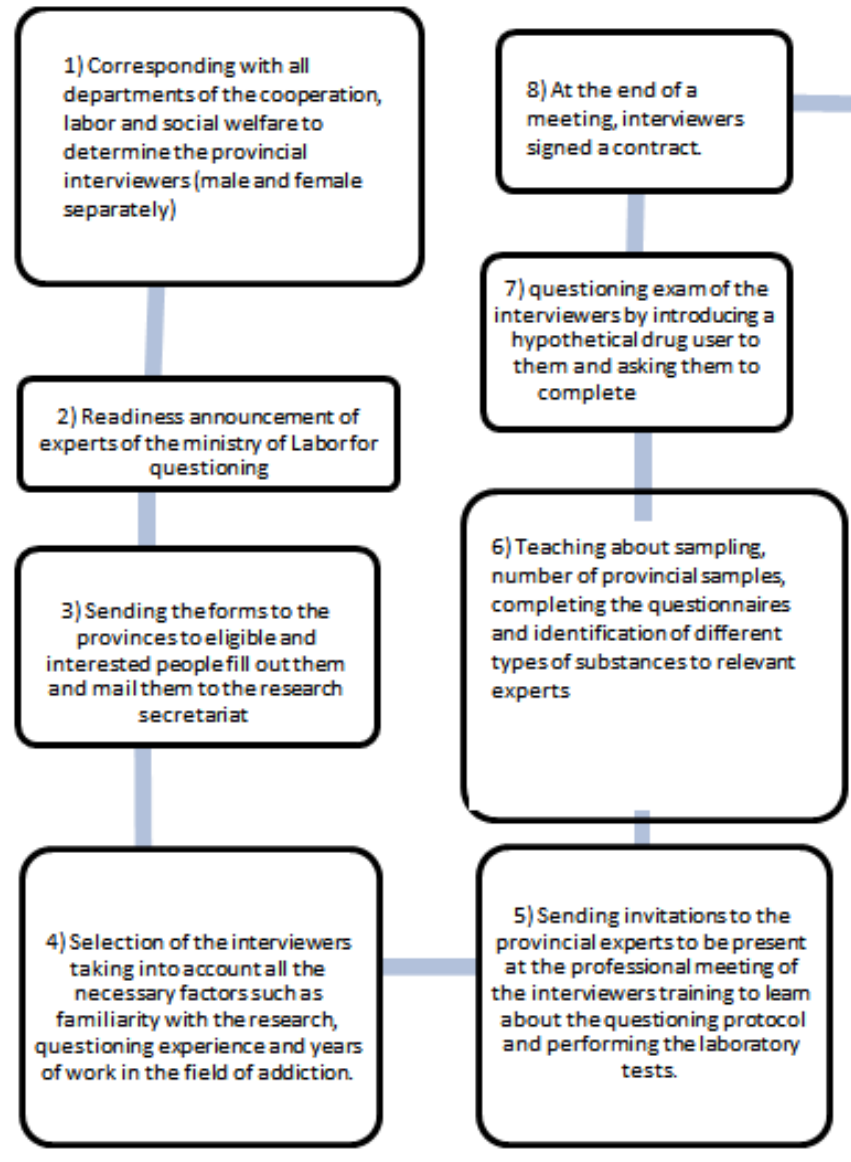

10) Each provincial expert posted the questionnaires filled out to the Executive Secretariat study after completion of $20 \%$ of their forms who participated in the project by the general director of the Ministry of Cooperatives, Labor and Social Welfare.

Fig. 1. Flowchart of selection, training, and evaluation of interviewers

Each sample test was transferred to a safe predicted place immediately. After completion of the sampling, all samples were assessed by 3 kits of hashish, methamphetamine, and morphine, and the results were written in the questionnaires. Those who had stress while doing the test and found it difficult to pass urine were asked to get some rest in the main room to relax. Doing the test was completely optional and those who refused to do the test were not obliged with force. Other research data on workplaces were accurately registered and reported (ie, results of the periodic tests or the experiences of sending people who were suspected of addiction to the laboratory as well as the status of using drugs in the past 72 hours that could have affected morphine urine test).

\section{Quality Control of the Fieldwork}

The study quality control was conducted in 2 levels

1. The Office of Research and Education of Headquarters for Combating Drugs had selected some observers for monitoring the process of the project implementation

2. The Ministry of Cooperatives, Labor, and Social Welfare established the research secretariat.

The colleagues in the secretariat (level 2) sent the reports of the project implementation to the first-level supervisors, with an ongoing reporting process. They also supervised the provincial interviewers who were reviewing and appraising the forms one by one after receiving the completed questionnaires sent by the provincial interviewers. Supervisors provided feedback to provincial representatives on how to fill out a questionnaire and perform laboratory tests in 2 stages:

At the first stage, $20 \%$ of the completed questionnaires were sent to the Secretariat by express post and were evaluated and defects were sought by scientific colleagues of the Secretariat. A separate letter was prepared for each province and the weakness points and key notifications were sent via email. At the second stage, the remaining questionnaires were sent to the Secretariat and, according to the guidelines, interviewers also sent the final report of their experiences, including barriers to enter the workplace, sampling, completion of the questionnaires, the impact of their gender on interviewing, testing, transportation, and their promotional proposals.

Iran DUWS (Drug Use in Workplaces Survey) instruments

In the present study, 2 instruments were used to collect data: (1) self-reported questionnaires, (2) laboratory 
diagnostic kits for verification.

1. Questionnaire: Objectives of the study were evaluated to design the instrument, and the national questionnaire was developed based on objectives of the survey. Pre-existing questionnaire of rapid assessment of drug use in Iran was selected as a framework to design the study questionnaire (12). The first section of the questionnaire was related to demographic questions. Then, the consumption patterns were questioned. International standards were also applied in this questionnaire,

meaning that consumption intervals during lifetime (ie, yearly, monthly, and current consumption) was questioned and different types of substances available in the market were included with expert consensus, including cannabis group (i.e., cannabis, weed or grass, flowers, and marijuana), opioids group (i.e., opium, opium sap, burned, burned sap, heroin, crack, and methadone-buprenorphine), group of glass and other substances (i.e., cocaine, narcotic pills (tramadol, codeine, and diphenoxylate), ecstasy, LSD, and other substances), except alcohol. The first and common consumptions were questioned. Time and place of drug use was also asked. Substance use in the family, the injection history, and crime commitment background were also incorporated in the questionnaire and, at the end, treatment history and its methods were questioned. In addition, there were several questions on the status of using drugs 72 hours before the test that could affect the morphine urine test, including stomach medicines, such as ranitidine, cimetidine, famotidine, omeprazole, and narcotic drugs, including tramadol, codeine, and diphenoxylate. The final question of the test was related to the urine test results. The cases with lack of cooperation or those who did not do the urine test were also mentioned in the questionnaire. The primary version of the designed questionnaire was sent to the observers to assess its face and content validity, and their comments were applied in the questionnaires. pharmaceutical factory in Mashhad was selected as a primary site to evaluate the validity and reliability of the questionnaire in the pilot phase. The Cronbach's alpha coefficient of the scales of the questionnaire ranged between 0.840 and 0959, showing the internal consistency of this instrument. Also, to assess the reliability of the questionnaire, the test-retest method was used and the workers evaluated in the pilot phase were asked to recomplete the questionnaires after 2 weeks. The intraclass correlation coefficient (ICC) calculation showed that the domains had an acceptable reliability. Moreover, most of the questionnaire domains had good reliability, with kappa 0.8 . The questionnaire was changed in domains that demonstrated poor or relatively poor reliability, and the final questionnaire was prepared for publication and forwarded to the interviewers.

2. Laboratory Kits: Rapid kits (serial numbers MOP3090034 and THC 3120018) were used for drug testing in this study, which have a relatively high sensitivity. In this method, glass, hashish, and its derivatives were traceable if being used up to 10 days prior to testing, and opium was recognizable if being used up to 4 days prior to testing, as these substances have high durability in the urine. Rapid kits can be used in an outpatient manner and results are immediately readable. Urine test comprised of dual-band of control and testing. The result was interpreted as positive if both bands were bold after testing and was interpreted as negative if just the control band was bold. The test was suspected if the control band was highlighted while the test band was lighter, and the test was not valid, if the control band did not get bold. The results of addiction urine tests were considered in 2 modes: optimistic and pessimistic. In the optimistic mode, just positive tests were considered as positive and others as negative, while in the pessimistic scenario, both positive and suspicious tests were considered as positive and others as negative.

\section{Ethical considerations}

Provincial interviewers were trained for the ethical

Table 3. Demographic characteristics and housing status of participants

\begin{tabular}{|c|c|c|c|}
\hline & & Number & Percent \\
\hline \multirow[t]{3}{*}{ Gender } & Male & 12077 & 92 \\
\hline & Female & 1051 & 8 \\
\hline & Total & 13128 & 100 \\
\hline \multirow[t]{5}{*}{ Age group (men) } & 20 years $\leq$ & 72 & 0.6 \\
\hline & 20-41 years & 8626 & 71.4 \\
\hline & 41-60 years & 3329 & 27.6 \\
\hline & 60 years $\geq$ & 50 & 0.4 \\
\hline & Total (men) & 12077 & 100 \\
\hline \multirow[t]{5}{*}{ Age group (women) } & 20 years $\leq$ & 35 & 3.3 \\
\hline & 20-41 years & 865 & 82.3 \\
\hline & $41-60$ years & 149 & 14.2 \\
\hline & 60 years $\geq$ & 2 & 0.2 \\
\hline & Total (women) & 1051 & 100 \\
\hline \multirow[t]{10}{*}{ Education level } & Illiterate & 158 & 1.2 \\
\hline & Read and write & 180 & 1.4 \\
\hline & Elementary school & 1322 & 10.1 \\
\hline & Guidance school & 2159 & 16.4 \\
\hline & High school & 954 & 7.3 \\
\hline & Diploma and pre university & 5240 & 39.9 \\
\hline & Fogh-e-Diplom & 1170 & 8.9 \\
\hline & Bachelor degree or higher & 1937 & 14.8 \\
\hline & etc. & 8 & 0.1 \\
\hline & Total & 13128 & 100 \\
\hline
\end{tabular}




\begin{tabular}{|c|c|c|c|}
\hline \multicolumn{4}{|l|}{ Table 3. Cntd } \\
\hline \multirow[t]{5}{*}{ Marital status } & Single & 2001 & 15.3 \\
\hline & Married & 11013 & 83.9 \\
\hline & Divorced & 99 & 0.7 \\
\hline & Widow & 15 & 0.1 \\
\hline & Total & 13128 & 100 \\
\hline \multirow[t]{4}{*}{ Number of children } & Without child & 2364 & 21 \\
\hline & 1-4 children & 8537 & 77 \\
\hline & 5 children or more & 226 & 2 \\
\hline & Total & 11127 & 100 \\
\hline \multirow[t]{5}{*}{ Religion } & Islam & 13099 & 99.7 \\
\hline & Zoroastrian & 10 & 0.1 \\
\hline & Christian & 9 & 0.1 \\
\hline & etc. & 10 & 0.1 \\
\hline & total & 13128 & 100 \\
\hline \multirow[t]{8}{*}{ Housing status } & Personal house ownership & 6245 & 47.6 \\
\hline & Rental house & 3763 & 28.7 \\
\hline & Parental house & 2919 & 22.2 \\
\hline & the organization homes & 167 & 1.3 \\
\hline & The home of relatives / friends & 19 & 0.1 \\
\hline & Gate keeper/ sentinel & 9 & 0.06 \\
\hline & homeless & 1 & 0.01 \\
\hline & etc. & 5 & 0.03 \\
\hline Total & & 13128 & 100 \\
\hline
\end{tabular}

considerations at the beginning of the study and they provided written commitment to comply with the ethical considerations during interviews. In addition, a comprehensive and detailed questioning instruction about moral issues was delivered to interviewers. To protect anonymity and confidentiality of samples' private information, other personals were asked to leave the room during the interview session. Interviewees were asked to give oral consent to participate in the study. A number was assigned to each person, which was written on the questionnaire and urine samples instead of participants' names. To respect the individual's rights and convenience, tests for female participants were taken by a female and those for males was done by a male. Participation in the study was voluntary and free and those who refused to give urine tests were not coerced into testing. Drug users who admitted their addiction were referred to detoxification centers. All questionnaires were submitted to the secretariat of the project, and data were kept confidential.

\section{Statistical analysis}

Study data were entered into the SPSS software and analyzed after reviewing for potential errors and ensuring data accuracy. Data analysis was performed by descriptive method. STATA software was used for the consolidated sample analysis to control possible bias due to different distribution of demographic characteristics of the participants. This potential bias was controlled by post stratification survey to ensure that the estimates obtained for each individual was considered as the representative of other Iranian workers in the same class. Table 3 shows the samples is in each stratum. In this study, response rate was not $100 \%$, however, this did not have any effect on the study analysis because this was a descriptive study. Descriptive statistics, such as frequency tables, were reported. Cluster analysis was applied to report statistics at province level and the country was divided into risk zones to compensate for the low sample size and strengthen the statistics at each zone.

\section{Results}

\section{Response rate}

The adjusted sample size for this survey was 13489 , of them $203(1.5 \%)$ were excluded due to lack of access or unwillingness to participate in the study. Finally, 13286 questionnaires were completed for the study participations, of which $13128(98.8 \%)$ were entered the final analysis, and $158(1.2 \%)$ were removed due to defects in the urinary drug testing. Most of the defects in urinary testing were in the provinces of Kermanshah, with $15 \%$ and Khuzestan with $13 \%$ deficit in urine tests (Table 4).

\section{Reliability of the results}

In the initial assessment of the questionnaires, it was revealed that many workers who had positive urine test did not express using drugs. This prompted the research team to run a test in 1 stage in Mazandaran province. Interviewers in this province were asking more detailed questions from employees with positive urine tests. Unfortunately, the results were not satisfactory, and it seemed that drug users were afraid of admitting their addiction in their workplace. Thus, it was not possible to recomplete these questionnaires. Therefore, the results of this section were underestimated, and the information about the substance types consumed by addicted employees was less valuable. However, data on estimates of the drug use prevalence was more reliable.

\section{Discussion}

\section{Strengths of the study design}

This study had several strengths. No other comprehensive study has been conducted in the country to examine the status of drug use in industrial environments. On the other hand, consumption trends in the industrial environment are not distinct from consumption trends in the society. Therefore, results of this study can be applied 
Table 4. Provincial distribution of the samples and the samples response rate

\begin{tabular}{|c|c|c|c|c|c|c|c|}
\hline \multirow{3}{*}{ Province } & \multirow{3}{*}{$\begin{array}{l}\text { Number of work- } \\
\text { shops }\end{array}$} & \multirow{2}{*}{\multicolumn{2}{|c|}{ Questionnaires }} & \multicolumn{4}{|c|}{ The status of the questionnaires } \\
\hline & & & & \multicolumn{2}{|c|}{ Analyzable } & \multicolumn{2}{|c|}{ Not Analyzable } \\
\hline & & Number & Percent & Number & Percent & Number & Percent \\
\hline Tehran & 113 & 2362 & 17.8 & 2352 & 99.6 & 10 & 0.4 \\
\hline Isfahan & 50 & 1244 & 9.4 & 1244 & 100 & 0 & 0 \\
\hline Razavi Khorasan & 41 & 863 & 6.5 & 861 & 99.8 & 2 & 0.2 \\
\hline Qazvin & 33 & 722 & 5.4 & 719 & 99.6 & 3 & 0.4 \\
\hline Alborz & 29 & 683 & 5.1 & 679 & 99.4 & 4 & 0.6 \\
\hline Markazi & 26 & 649 & 4.9 & 648 & 99.8 & 1 & 0.2 \\
\hline East Azarbaijan & 30 & 628 & 4.7 & 626 & 99.7 & 2 & 0.3 \\
\hline Khuzestan & 28 & 601 & 4.5 & 522 & 87 & 79 & 13 \\
\hline Mazandaran & 23 & 432 & 3.3 & 430 & 99.5 & 2 & 0.5 \\
\hline Fars & 20 & 425 & 3.2 & 424 & 99.8 & 1 & 0.2 \\
\hline Yazd & 18 & 395 & 3.0 & 395 & 100 & 0 & 0 \\
\hline Gilan & 18 & 379 & 2.9 & 379 & 100 & 0 & 0 \\
\hline Semnan & 15 & 302 & 2.3 & 302 & 100 & 0 & 0 \\
\hline Zanjan & 13 & 255 & 1.9 & 254 & 99.6 & 1 & 0.4 \\
\hline Qom & 12 & 233 & 1.8 & 230 & 98.7 & 3 & 1.3 \\
\hline Hormozgan & 11 & 224 & 1.7 & 224 & 100 & 0 & 0 \\
\hline South Khorasan & 9 & 203 & 1.5 & 203 & 100 & 0 & 0 \\
\hline Bushehr & 5 & 200 & 1.5 & 200 & 100 & 0 & 0 \\
\hline Ilam & 10 & 200 & 1.5 & 200 & 100 & 0 & 0 \\
\hline Sistan and Baluchestan & 7 & 200 & 1.5 & 200 & 100 & 0 & 0 \\
\hline Kerman & 10 & 200 & 1.5 & 182 & 91 & 18 & 9 \\
\hline Kermanshah & 9 & 200 & 1.5 & 170 & 85 & 30 & 15 \\
\hline Kohgiluye and Boyer-Ahmad & 7 & 200 & 1.5 & 200 & 100 & 0 & 0 \\
\hline Chaharmahal and Bakhtiari & 10 & 200 & 1.5 & 200 & 100 & 0 & 0 \\
\hline West Azarbaijan & 10 & 200 & 1.5 & 199 & 99.5 & 1 & 0.5 \\
\hline North Azarbaijan & 7 & 198 & 1.5 & 198 & 100 & 0 & 0 \\
\hline Lorestan & 10 & 195 & 1.5 & 195 & 100 & 0 & 0 \\
\hline Kordestan & 9 & 194 & 1.5 & 194 & 100 & 0 & 0 \\
\hline Ardebil & 9 & 177 & 1.3 & 177 & 100 & 0 & 0 \\
\hline Hamedan & 9 & 165 & 1.2 & 165 & 100 & 0 & 0 \\
\hline Golestan & 8 & 157 & 1.2 & 156 & 99.4 & 1 & 0.6 \\
\hline Total & 609 & 13286 & 100.0 & 13128 & 98.8 & 158 & 1.2 \\
\hline
\end{tabular}

as a document for policymakers to design the needed interventions in the workplace and to decide on the needed measurements for the community. One of the positive features of this study was reliability of results on the prevalence of drug use, which was consistent with the obtained results of Asalouyeh (9). However, the present survey was implemented in a wider scale and at the national level. This study had also higher power in comparison with the study of Asalouyeh in better quality of measurement method, as in this study, urine test was used for verification of self-reported questionnaires. In addition, data collection was done with rigorous training and supervision of interviewers, and fieldwork was done with high standards. At least 20 meetings were held by the project executive and his colleagues to improve the quality of data collection. Therefore, unresponsiveness was minimized and $98.8 \%$ of the collected data were complete and analyzable, and those who had refused to give urine test constituted only a small fraction of the participants $(1.2 \%)$.

\section{Limitations of the study}

This study had some limitations. First, those who were responding to the questionnaires, sometimes, did not express a history of drug use due to fear of job loss or did not honesty talk about their history of alcohol consumption for cultural reasons. Stomach drugs, such as ranitidine, cimetidine, famotidine, omeprazole, or drugs, such as tramadol, codeine, and diphenoxylate, may alter the results of urine tests for drug addiction if used up to 72 hours prior to testing. Some of the employees refused to do the urine test. Although the questionnaire results were negative in some cases, the urine test revealed positive results in the same people. In such situations, questioning was repeated, but these employees did not complete the questionnaires again. Hair analysis is suggested as a biological indicator for addiction in some studies that have been successful in correct identification of drug users. The problems mentioned above could be solved in case of using hair analysis (13), but this method was not used in this study due to its high cost.

There were also several other limitations at the beginning of the project, such as difficulty of the interviewers to enter workshops and coordinating with the employers. Moreover, most of the samples did not cooperate with the interviewers at first. These limitations were facilitated after sending a letter to employers and coordinating with the head of the workshop.

\section{Conclusion}

The present report reviews the protocol and methodology of the national survey of prevalence of addiction in the country's industrial environments. The survey results are reliable because of the high response rate and the verification samples' statements in the questionnaire with the drug urine test. Interventions to reduce addiction in industrial environments can be costeffective. Thus, the results of this national survey could 
be used for seeking support from authorities. Policymakers can apply the results of this study to plan for prevention of addiction in industrial environments by creating staff cooperative units.

\section{Acknowledgements}

The study was financially supported by Research and Education Office of Headquarters for Combating Drugs. The authors acknowledge supports from Dr. Hooman Narenjiha, Dr. Iraj Ismaeeli, Dr. Farshid Alaedini, Dr. Hamid Sarrami, and Dr. Gholamreza Bostanmanesh. The authors would also like to thank provincial field interviewers as well as all the participants in the study.

\section{Conflict of Interests}

The authors declare that they have no competing interests.

\section{References}

1. Carter M, McGee R, Taylor B, Williams S. Health outcomes in adolescence: Associations with family, friends and school engagement. J Adolesc. 2007; 30(1):51-62.

2. Khazaei S, Mohammadian-Hafshejani A, Ahmadi Pishkuhi M, Salehinia H. Proportion of mortality attributable to tobacco worldwide. Iran J Public Health. 2016;45(3):399-400.

3. Ehterami M. Conformity of Drug Abuse International Standards, Psychological and Sociologic Theories and Drug and Alcohol Use Prevention in Iran in 4 National Development Programs. Asia Pac J Med Toxicol. 2014;3:21-21.

4. Narenjiha H, Rafiei H, Noori R, Shirinbayan P, Farhadi MH, Etemadi H. Rapid assessment of drug abuse in Iran. Tehran: Danjeh, 2009.

5. Khazaei S, Poorolajal J, Mahjub H, Esmailnasab N, Mirzaei M. Estimation of the frequency of intravenous drug users in Hamadan city, Iran, using the capture-recapture method. Epidemiol Health. 2012;34:1-6.

6. Khorshidi A, Moradi A, Shakiba M, Rahmani Kh. Estimating the prevalence of drug abuse in ilam using capture-recapture method. JMUMS. 2015;25(124):194-199.

7. Nikfarjam A, Shokoohi M, Shahesmaeili A, Haghdoost AA, Baneshi MR, Haji-Maghsoud S, et al. National population size estimation of illicit drug users through the network scale-up method in 2013 in Iran. Int. J. Drug Policy. 2016;31:147-152.

8. Carpenter CS. Workplace drug testing and worker drug use. Health Serv Res. 2007;42(2):795-810.

9. Saberi-Zafarghandi MB, Rahimi-Movaghar A, Hajebi A, AminEsmaeili M, Razaghi EM, Khastoo G, et al. Situation of Drug Use, Addiction and Related Services in the Laborers of Assaluyeh Industrial Area (South Pars Gas Field) in 2007. Hakim Res J. 2010;12(4):58-68.

10. Mokri A. Brief overview of the status of drug abuse in Iran. Arch Iran Med. 2002;5(3):184-190.

11. Damari B, Ahmadi-Pishkuhi M, Masoudiasl I, Bostanmanesh G. Interventions to Reduce Drug Abuse in Pars Special Economic Energy Zone. Iran Red Crescent Med J. 2015; 17(11):1-9.

12. Razzaghi E, Rahimi A, Hosseini M, Madani S. Rapid Situation Assessment (RSA) of drug abuse in Iran. Prevention Department, State Welfare Organization, Ministry of Health, IR of Iran and United Nations International Drug Control Program, 1999.

13. Cook RF, Bernstein AD, Andrews CM. Assessing drug use in the workplace: a comparison of self-report, urinalysis, and hair analysis. NIDA Res Monogr. 1997;167(247):72. 